\title{
Interpolação de perfis radiais de distribuição de água de aspersores
}

\author{
Giuliani do Prado $^{1}$ \& Alberto Colombo ${ }^{2}$
}

\begin{abstract}
RESUMO
Neste estudo é apresentado e avaliado um procedimento para determinar perfis radiais de distribuição de água em condições operacionais intermediárias àquelas dos ensaios. Utilizaram-se 40 perfis radiais do aspersor PLONA-RL250 obtidos com diferentes combinações de 8 diâmetros do bocal principal e 5 pressões de serviço. Perfis radiais medidos nas pressões de 294, 490 e $686 \mathrm{kPa}$ foram utilizados para reproduzir perfis nas pressões de 392 e $588 \mathrm{kPa}$. Perfis obtidos com bocais de $14 \times 6,18 \times 6,22 \times 6$ e $26 \times 6 \mathrm{~mm}$ foram utilizados para reproduzir os perfis com bocais de $16 \times 6,20 \times 6$ e $24 \times 6 \mathrm{~mm}$. Os perfis radiais gerados para pressão de serviço e bocais, comparados aos perfis observados, respectivamente, apresentaram valores médios de: i) coeficiente de determinação de 0,921 e 0,921; ii) coeficiente de variação de 9,5 e 9,4\%; iii) probabilidade (teste t de Student) de 88,5 e $87,3 \%$. Simulações digitais da uniformidade de aplicação de água a partir dos perfis radiais observados e gerados por interpolação entre pressão de serviço e diâmetro de bocais apresentaram, respectivamente, erros absolutos médios de 1,36 e 1,44\%. O procedimento proposto não influencia significativamente os valores de uniformidade de aplicação de água.
\end{abstract}

Palavras-chave: uniformidade de irrigação, diâmetro de bocal, pressão de trabalho

\section{Sprinkler radial water distribution profiles interpolation}

\begin{abstract}
A procedure is described to determine and evaluate radial water distribution profiles for a sprinkler head operating at conditions intermediate to those of the tests. Forty water distribution profiles from a PLONARL250 sprinkler, obtained under different combinations of 8 nozzle diameters and 5 working pressures, were used. Profiles determined under pressures of 294, 490, $686 \mathrm{kPa}$ were used to generate profiles for 392 and $588 \mathrm{kPa}$ pressures. Profiles determined for $14 \times 6,18 \times 6,22 \times 6$ and $26 \times 6 \mathrm{~mm}$ nozzle diameters were used to reproduce radial profiles for $16 \times 6,20 \times 6$ and $24 \times 6 \mathrm{~mm}$ nozzle diameters. The radial profiles generated for working pressure and nozzle diameters, compared to the radial profiles observed, respectively, had mean values of: i) determination coefficient of 0.921 and 0.921 ; ii) coefficient of variation of 9.5 and $9.4 \%$; iii) probability (Student $t$ test) of 88.5 and $87.3 \%$. Computer simulations of water application uniformity from radial profiles observed and generated for working pressure and nozzle diameters presented, respectively, mean absolute deviation of 1.36 and $1.44 \%$. The procedure proposed does not influence significantly the values of water application uniformity.
\end{abstract}

Key words: irrigation uniformity, nozzle size, working pressure 


\section{INTRODUÇÃO}

Face à alta competitividade e às exigências do mercado, os setores de produção agrícola buscam cada vez mais produzir bens de consumo com alta eficiência técnica e econômica; entretanto, o incremento produtivo em quantidade e qualidade visando reduzir os custos de produção, está atrelado a estratégias de proteção ambiental, principalmente ao uso e à conservação dos recursos hídricos.

Devido às exigências hídricas da cultura e ao cultivo em grandes extensões de terra, a produção agrícola sob irrigação é um dos setores da cadeia produtiva que mais consomem água. Desta forma, sistemas de irrigação bem dimensionados, que apresentem índices adequados de uniformidade de aplicação de água, aliados a um manejo correto da irrigação, são essenciais para maximizar a produtividade e reduzir as perdas de água (Prado \& Colombo, 2011; Oliveira et al., 2012).

Os sistemas de irrigação são projetados de modo que a aplicação de água seja a mais uniforme possível sendo que em cada método de irrigação recomenda-se um valor mínimo de uniformidade de aplicação de água. No caso da aspersão é aconselhável que os valores de uniformidade (coeficiente de Christiansen - CUC) sejam superiores a 80\% (Bernardo et al., 2005). De acordo com Álvarez et al. (2004), altos valores de uniformidade de aplicação de água são fundamentais para a obtenção de um retorno adequado econômico da cultura pois se consegue, assim, maior produtividade por unidade de área com um consumo menor de água, energia e fertilizantes.

Em razão dos inúmeros fatores que influenciam na uniformidade de aplicação de água de um sistema de irrigação por aspersão (tipo de aspersor, pressão de serviço, disposição dos aspersores e condições de vento), a realização de ensaios de campo torna-se uma tarefa demorada e, na maioria das vezes, não se consegue realizar as avaliações em todas as condições operacionais e ambientais desejadas (Carrión et al., 2001; Oliveira et al., 2009; Faria et al., 2009; 2012).

Para facilitar o processo de análise da uniformidade de aplicação de água em diferentes condições operacionais do sistema de irrigação, o uso de simulações computacionais torna-se uma ferramenta extremamente útil devido à agilidade, à precisão e à segurança dos resultados obtidos. Deste modo, as simulações assumem importante papel na tomada de decisão em projetos de irrigação, na avaliação de equipamentos de irrigação, no desenvolvimento de novos aspersores ou para avaliar se procedimentos usualmente adotados são adequados (Prado \& Colombo, 2010a).

No caso específico dos sistemas de irrigação por aspersão, as simulações digitais são efetuadas a partir dos perfis estacionários de distribuição de água dos aspersores operando nas mesmas condições de campo (Vories et al., 1987; Carrión et al., 2001; Prado \& Colombo, 2010b). Desta forma, esses modelos requerem que se determinem as características de distribuição de água (perfil radial) dos diversos modelos de aspersores operando em diferentes combinações de pressão de serviço e diâmetro de bocais.

A existência de inúmeras possibilidades de combinações de pressão de serviço e diâmetro de bocais e a dificuldade para realização de ensaios de distribuição de água, principalmente quando os ensaios são de aspersores de grande porte (canhões hidráulicos), tornam necessários procedimentos que permitam determinar perfis radiais de distribuição de água de aspersores em condições operacionais intermediárias àquelas ensaiadas em laboratórios (Prado \& Colombo, 2009).

Alguns procedimentos de interpolação do perfil radial de distribuição de água para condições operacionais não ensaiadas são apresentados na literatura. Louie \& Selker (2000) se referem a um procedimento de interpolação no qual o perfil radial do aspersor, ensaiado com um diâmetro de bocal menor mas para a mesma pressão de serviço, é usado para determinar o perfil radial do aspersor operando com um bocal maior. Neste procedimento os pares de valores de distância ao aspersor e intensidade de precipitação são multiplicados pela razão entre a vazão do aspersor na condição a ser interpolada e a vazão na condição em que o perfil radial foi determinado.

Solomon \& Bezdek (1980) apresentam um procedimento de interpolação cujas diferentes formas geométricas típicas assumidas pelo perfil radial de distribuição de água em função de diferentes combinações de bocais e pressão de serviço, são utilizadas para determinar o perfil radial para condições operacionais não ensaiadas.

Apesar da importância dessas interpolações não existe, até o momento, um procedimento padrão. Neste trabalho é apresentada uma metodologia de interpolação dos valores de intensidade de precipitação de perfis radiais adimensionais de aspersores operando em condições intermediárias àquelas ensaiadas. A metodologia apresentada une os perfis radiais adimensionais propostos por Solomon \& Bezdek (1980) ao simples processo de interpolação demonstrado por Louie \& Selker (2000).

\section{Material e Métodos}

O trabalho foi desenvolvido utilizando-se quarenta perfis radiais de distribuição de água de um aspersor PLONA-RL250 determinados seguindo a metodologia descrita por Prado \& Colombo (2005). Os perfis radiais de distribuição de água e os valores de vazão e raio de alcance, foram determinados com o aspersor operando com oito diâmetros de bocais diferentes (14 x 6,16 × $6,18 \times 6,20 \times 6,22 \times 6,24 \times 6,26 \times 6$ e $28 \times 6$ mm) em cinco pressões de serviço $(294,392,490,588$ e $686 \mathrm{kPa})$.

Cada perfil radial de distribuição de água foi adimensionalizado seguindo a metodologia apresentada por Solomon \& Bezdek (1980). Desta forma e conforme as Eqs. 1 e 2, os valores de distância ao aspersor $\left(r_{i}\right)$ foram expressos em termos da fração $\left(\mathrm{ra}_{\mathrm{i}}\right)$ do raio de alcance $(\mathrm{R})$ e os valores de intensidade de precipitação $\left(i_{i}\right)$ em termos da fração $\left(i a_{i}\right)$ da intensidade média de aplicação de água (im).

$$
\begin{gathered}
\mathrm{ra}_{\mathrm{i}}=\frac{\mathrm{r}_{\mathrm{i}}}{\mathrm{R}} \\
\mathrm{ia}_{\mathrm{i}}=\frac{\mathrm{i}_{\mathrm{i}} \cdot \pi \cdot \mathrm{R}^{2}}{\mathrm{Q}}=\frac{\mathrm{i}_{\mathrm{i}}}{\mathrm{im}}
\end{gathered}
$$

em que: 

$\mathrm{ra}_{\mathrm{i}} \quad$ - fração do raio de alcance
$r_{i} \quad$ - distância ao aspersor, $m$
$\mathrm{R}$ - raio de alcance, $\mathrm{m}$
$\mathrm{ia}_{\mathrm{i}} \quad$ - fração da intensidade média de aplicação de água
$\mathrm{i}_{\mathrm{i}} \quad$ - intensidade de precipitação, $\mathrm{m} \mathrm{h}^{-1}$
im - intensidade média de aplicação de água, $\mathrm{m} \mathrm{h}^{-1}$
$\mathrm{Q} \quad$ - vazão do aspersor, $\mathrm{m}^{3} \mathrm{~h}^{-1}$
i - índice de identificação de cada ponto de amostragem (coletor) em relação ao aspersor

As informações contidas em cada perfil radial adimensional de distribuição de água foram reduzidas para apenas vinte valores de intensidade de precipitação de água (ia ${ }_{\mathrm{j}}$ ), correspondentes às distâncias adimensionais dadas pela Eq. 3 .

$$
\mathrm{ra}_{\mathrm{j}}=0,025+(\mathrm{j}-1) \cdot 0,05 \quad \text { com } 20 \geq \mathrm{j} \geq 1
$$

Com os perfis radiais adimensionais procedeu-se a uma interpolação linear, dos valores de intensidade de precipitação adimensional (ia ), dados pelas distâncias adimensionais $\mathrm{ra}_{\mathrm{j}}$, entre dois intervalos de pressão de serviço, conforme a Eq. 4. O mesmo procedimento foi efetuado para interpolar os valores de intensidade de precipitação adimensional (ia $)$, dados pelas distâncias adimensionais $\mathrm{ra}_{\mathrm{j}}$, entre dois intervalos de bocal principal, conforme a Eq. 5.

$$
\begin{aligned}
& \operatorname{iad}_{j}=\frac{\operatorname{ias}_{\mathrm{j}}(p d-p i)+\operatorname{iai}_{\mathrm{j}}(p s-p d)}{p s-p i} \quad \operatorname{com} 20 \geq j \geq 1 \\
& \operatorname{iad}_{\mathrm{j}}=\frac{\operatorname{ias}_{\mathrm{j}}(\mathrm{bd}-\mathrm{bi})+\mathrm{iai}_{\mathrm{j}}(\mathrm{bs}-\mathrm{bd})}{\mathrm{bs}-\mathrm{bi}} \quad \operatorname{com} 20 \geq \mathrm{j} \geq 1
\end{aligned}
$$

em que:

iad $_{j}$ - intensidade de precipitação adimensional obtida por interpolação para a pressão desejada ( $\mathrm{pd}, \mathrm{kPa}$ ) ou para o bocal desejado (bd, $\mathrm{mm}$ )

ias $_{j}$ - intensidade de precipitação adimensional do perfil radial adimensional obtido na pressão imediatamente superior (ps, kPa) à pressão desejada ou para o bocal imediatamente acima (bs, mm) ao bocal desejado

iai $_{j} \quad$ - intensidade de precipitação adimensional do perfil radial adimensional obtido na pressão imediatamente inferior (pi, $\mathrm{kPa}$ ) à pressão desejada ou para o bocal imediatamente abaixo (bi, mm) ao bocal desejado

O processo de interpolação proposto foi aplicado para reproduzir, nos oito diâmetros de bocais ensaiados, os valores adimensionais de intensidade de precipitação correspondentes às pressões de 394 e $588 \mathrm{kPa}$ utilizando-se informações dos perfis observados, respectivamente, nas pressões de 294 e 490 $\mathrm{kPa}$ e 490 e $686 \mathrm{kPa}$. Testou-se, ainda, o processo proposto para reproduzir, nas cinco pressões ensaiadas, os valores adimensionais de intensidade de precipitação correspondentes aos bocais de 16 × 6;20 x 6 e 24 x 6 mm com base nos perfis observados, respectivamente, com bocais $14 \times 6$ e $18 \times 6 \mathrm{~mm}$; 18 × 6 e 22 × 6 mm; e 22 × 6 e 26 × 6 mm.

Para a utilização dos perfis radiais adimensionais de distribuição de água em simulações computacionais, é notória a necessidade de transformá-los na sua forma dimensional a partir dos valores de vazão e raio de alcance do aspersor. Desta forma, os valores de vazão e raio de alcance obtidos em laboratório em função da pressão de serviço e do diâmetro do bocal principal do aspersor, foram utilizados para gerar os parâmetros de ajuste (Eqs. 6 e 7); neste procedimento utilizou-se o método dos mínimos quadrados empregando-se a ferramenta solver na planilha eletrônica Excel.

$$
\begin{gathered}
Q=k \cdot b^{x} \cdot p^{y} \\
R=a_{1} p^{2}+a_{2} p+a_{3} p \cdot b+a_{4} b^{2}+a_{5} b+a_{6}
\end{gathered}
$$

em que:

p - pressão de serviço, $\mathrm{kPa}$

b - diâmetro do bocal principal, $\mathrm{mm}$

k, x, y - parâmetros de ajuste da equação de vazão

$a_{1}, a_{2}, a_{3}, a_{4}, a_{5}, a_{6}$ - parâmetros de ajuste da equação de raio de alcance do aspersor

Em função do processo de interpolação dos perfis radiais adimensionais de distribuição de água entre pressões de serviço e entre bocais do aspersor, os ajustes dos parâmetros das equações de vazão e raio de alcance foram gerados para três condições distintas:

i) ajuste com todos os 40 valores de vazão ou raio de alcance determinados nos ensaios.

ii) ajuste desconsiderando 16 valores de vazão ou raio de alcance correspondente às interpolações a serem feitas (pressões de 394 e $588 \mathrm{kPa}$ em 8 bocais diferentes).

iii) ajuste desconsiderado 15 valores de vazão ou raio de alcance correspondente às interpolações com bocais de $16 \times 6$, $20 \times 6$ e $24 \times 6$ mm nas cinco pressões de serviço consideradas $(294,392,490,588$, e $686 \mathrm{kPa})$.

De modo a verificar a aderência dos 20 valores de intensidades de precipitação adimensionais dos perfis radiais gerados por interpolação, entre pressões de serviço e bocais, aos 20 valores de intensidades de precipitação adimensionais determinados em laboratório para cada perfil radial obtido, foram efetuadas, com auxílio da planilha eletrônica Excel, as seguintes análises: i) coeficiente de determinação $\left(\mathrm{R}^{2}\right)$; ii) coeficiente de variação $(\mathrm{CV})$ calculado pela razão entre o desvio padrão das diferenças entre os valores de intensidades de precipitações adimensionais observadas e interpoladas pela média dos valores de intensidade de precipitação adimensionais observadas; iii) probabilidade, pelo teste t de Student, dos valores de intensidades de precipitações adimensionais observadas e interpoladas serem iguais.

A aderência dos valores de intensidade de precipitação dos perfis radiais obtidos por interpolação aos valores de intensidade de precipitação dos perfis radiais determinados em laboratório, também foi avaliada através da análise da uniformidade de aplicação de água de equipamentos autopropelidos de irrigação.

Desenvolveu-se, para esta análise, um aplicativo computacional em Visual Basic da planilha eletrônica Excel, seguindo modelo descrito por Prado (2004). A partir deste programa foram gerados os valores de uniformidade de 
aplicação de água, coeficiente de Christiansen (CUC), com os perfis radiais interpolados e pelos perfis radiais determinados em laboratório. Conforme as especificações de operação de sistemas autopropelidos de irrigação apresentadas por Rocha et al. (2005) considerou-se o aspersor deslocando-o ao longo do carreador com uma velocidade linear constante de $50 \mathrm{~m} \mathrm{~h}^{-1}$, operando com um ângulo de giro de $270^{\circ}$ e para espaçamentos de carreadores compreendidos entre 40 a $80 \%$ do diâmetro molhado do aspersor.

Os valores de uniformidade de Christiansen obtidos com os perfis radiais determinados em laboratório foram relacionados aos valores de uniformidade de Christiansen obtidos com os perfis radiais interpolados, a partir de uma equação de reta ajustada; determinou-se, também, o erro absoluto entre os valores de uniformidade de Christiansen.

\section{Resultados E Discussão}

O efeito do número de ensaios considerados sobre o valor dos parâmetros das equações de vazão e raio de alcance do aspersor é mostrado nos dois grupos de equações (Eqs. 8, 9 e 10, e Eqs. 11, 12 e 13).

Na primeira equação de cada grupo (Eqs. 8 e 11) foram considerados todos os 40 valores de vazão ou raio de alcance determinados nos ensaios. Na segunda equação de cada grupo (Eqs. 9 e 12) foram desconsiderados 16 valores de vazão ou raio de alcance correspondentes às interpolações (pressões de 394 e $588 \mathrm{kPa}$ em 8 bocais diferentes). Na terceira equação de cada grupo (Eqs. 10 e 13) foram desconsiderados 15 valores de vazão ou raio de alcance, correspondentes aos perfis radiais com bocais de $16 \times 6,20 \times 6$ e 24 × $6 \mathrm{~mm}$ nas cinco pressões consideradas $(294,392,490,588$ e $686 \mathrm{kPa})$.

Observa-se, para os dois grupos de equações de vazão e de raio de alcance, que os valores dos coeficientes de determinação foram todos superiores a $99 \%$ revelando um bom ajuste aos dados observados nos ensaios de laboratório. Conforme a ordem relacionada acima, as equações de vazão (Q) são dadas por:

$$
\begin{array}{ll}
\mathrm{Q}=0,00846 \times \mathrm{b}^{1,7613} \times \mathrm{p}^{0,4937} & \left(\mathrm{R}^{2}=0,999\right) \\
\mathrm{Q}=0,00844 \times \mathrm{b}^{1,7618} \times \mathrm{p}^{0,4940} & \left(\mathrm{R}^{2}=0,999\right) \\
\mathrm{Q}=0,00788 \times \mathrm{b}^{1,7891} \times \mathrm{p}^{0,4922} & \left(\mathrm{R}^{2}=0,999\right)
\end{array}
$$

As equações de raio de alcance (R), conforme a ordem relacio-nada, são dadas por:

$$
\begin{aligned}
\mathrm{R} & =0,00006 \mathrm{p}^{2}+0,056 \mathrm{p}+0,0023 \mathrm{pb} \times \\
& \times 0,066 \mathrm{~b}^{2}+2,586 \mathrm{~b} \times 15,010 \quad\left(\mathrm{R}^{2}=0,999\right) \\
\mathrm{R} & =0,00006 \mathrm{p}^{2}+0,060 \mathrm{p}+0,0022 \mathrm{pb} \times \\
& \times 0,062 \mathrm{~b}^{2}+2,474 \mathrm{~b} \times 15,018 \quad\left(\mathrm{R}^{2}=0,996\right)
\end{aligned}
$$

$$
\begin{aligned}
\mathrm{R} & =0,00006 \mathrm{p}^{2}+0,053 \mathrm{p}+0,0024 \mathrm{pb} \times \\
& \times 0,081 \mathrm{~b}^{2}+3,147 \mathrm{~b} \times 19,946 \quad\left(\mathrm{R}^{2}=0,997\right)
\end{aligned}
$$

Nas Figuras 1 e 2 são apresentados perfis radiais adimensionais de distribuição de água obtidos em laboratório (linha azul) e os perfis radiais adimensionais de distribuição de água gerados através do procedimento de interpolação (linha vermelha) entre pressões de serviço (Figura 1) ou entre bocais principais do aspersor (Figura 2). Nessas figuras verifica-se que os valores de intensidade de precipitação adimensionais obtidos em laboratório e gerados pelo processo de interpolação são bastante próximos.
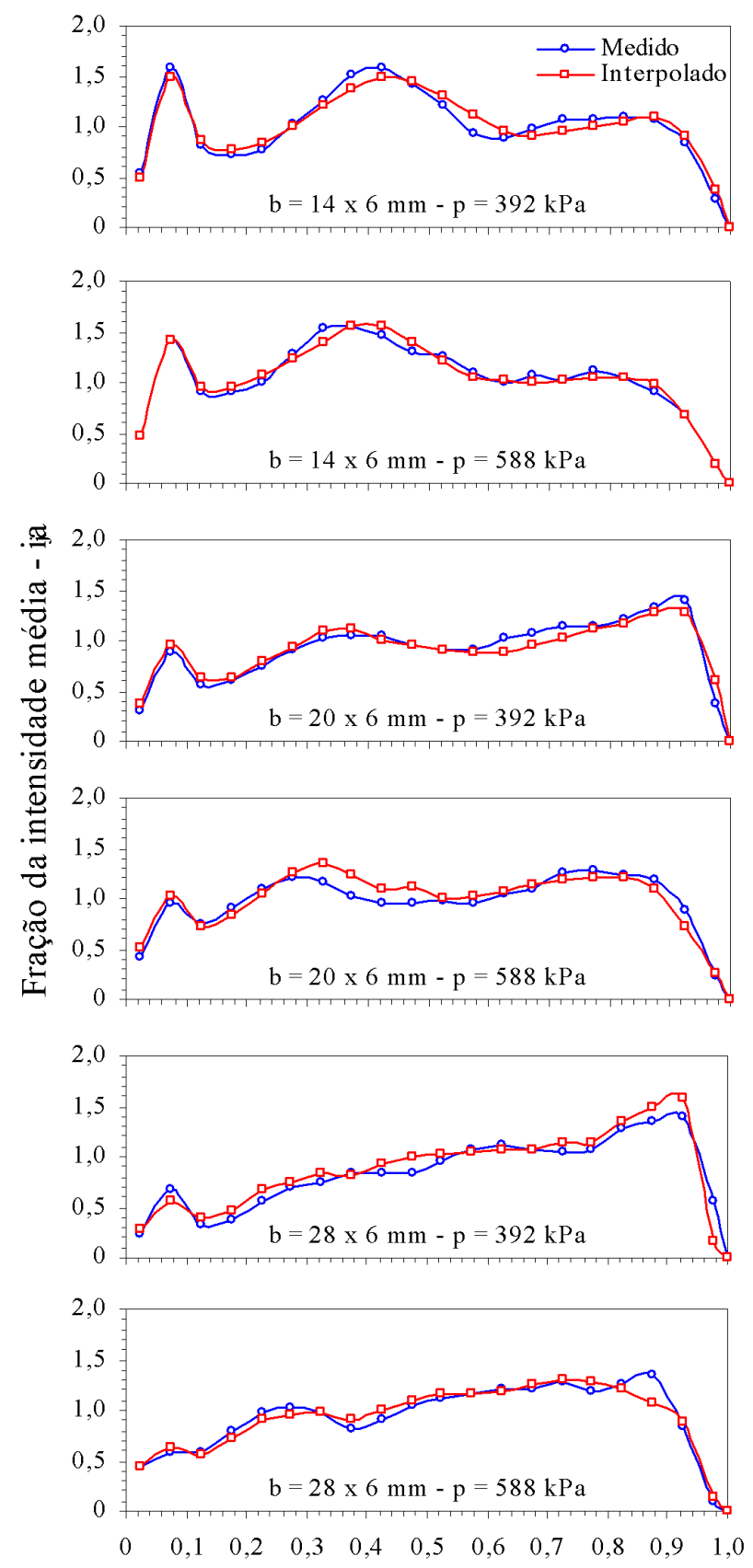

Fração do raio de alcance - $\mathrm{ra}_{\mathrm{j}}$

Figura 1. Perfis radiais adimensionais observados e gerados por interpolação entre pressões de serviço 

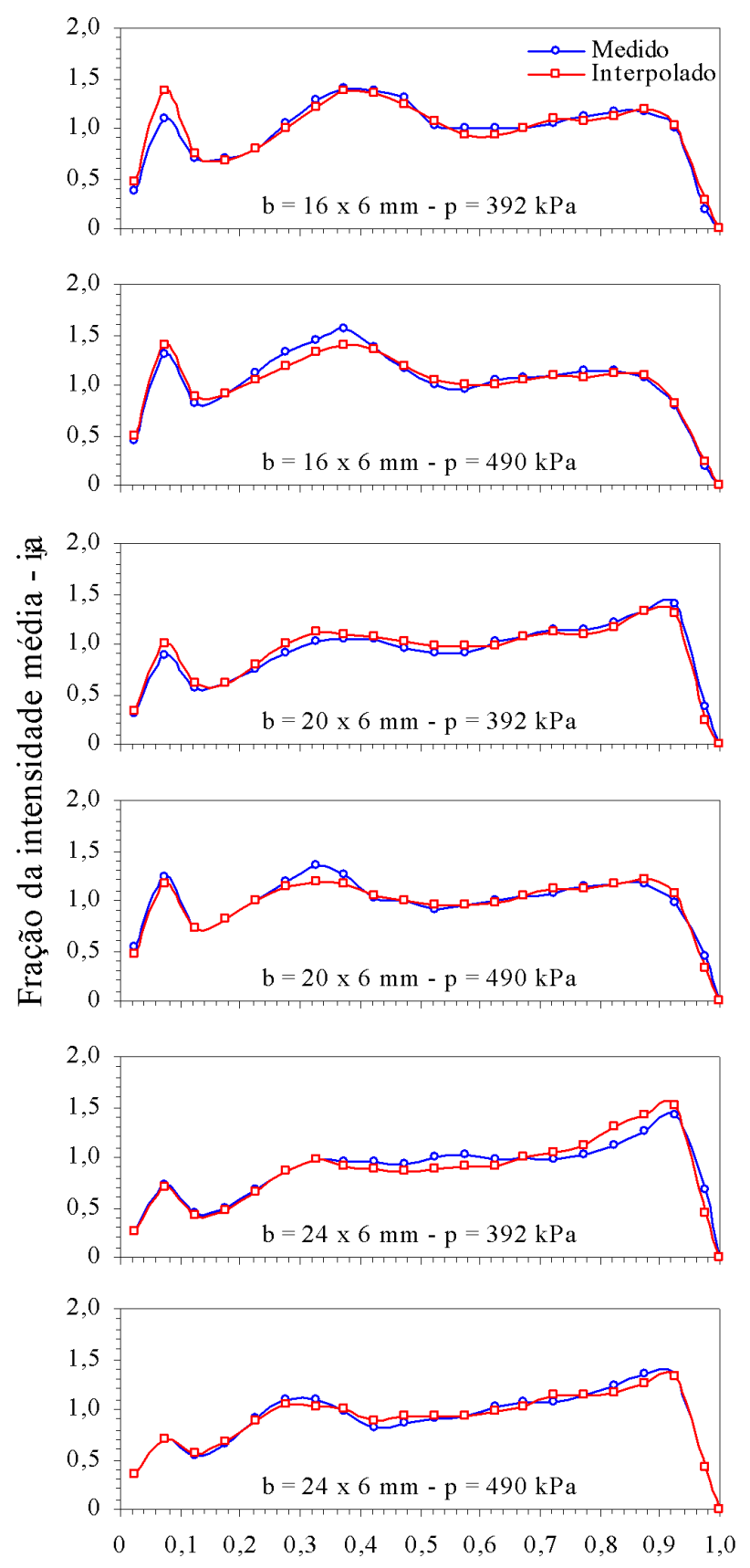

Fração do raio de alcance - $\mathrm{ra}_{\mathbf{j}}$

Figura 2. Perfis radiais adimensionais observados e gerados por interpolação entre bocais

Nas Tabelas 1 e 2 verifica-se, na comparação entre os perfis obtidos em laboratório e gerados por interpolação, que os valores médios de coeficiente de determinação foram todos superiores a $90 \%$. Molle \& Gat (2000) consideraram, na validação do seu modelo de simulação, que valores de coeficiente de determinação maiores que 0,85 são adequados para validar um modelo.

Tabela 1. Valores de coeficientes de determinação $\left(R^{2}\right)$, coeficientes de variação $(C V)$ e probabilidades de igualdade $(\mathrm{P}(\mathrm{t}))$, pelo teste t de Student, observados nas interpolações do perfil radial entre pressões de serviço

\begin{tabular}{|c|c|c|c|c|c|c|}
\hline \multirow{3}{*}{$\begin{array}{l}\text { Bocais } \\
(\mathrm{mm})\end{array}$} & \multicolumn{6}{|c|}{ Pressão de serviço $(\mathrm{kPa})$} \\
\hline & \multicolumn{3}{|c|}{392} & \multicolumn{3}{|c|}{588} \\
\hline & $\mathbf{R}^{2}$ & CV & $P(t)$ & $\mathbf{R}^{2}$ & CV & $P(t)$ \\
\hline $14 \times 6$ & 0,939 & 0,086 & 0,981 & 0,971 & 0,055 & 0,982 \\
\hline $16 \times 6$ & 0,973 & 0,058 & 0,912 & 0,952 & 0,071 & 0,944 \\
\hline $18 \times 6$ & 0,964 & 0,055 & 0,879 & 0,905 & 0,096 & 0,800 \\
\hline $20 \times 6$ & 0,932 & 0,096 & 0,950 & 0,867 & 0,102 & 0,761 \\
\hline $22 \times 6$ & 0,830 & 0,139 & 0,810 & 0,896 & 0,100 & 0,940 \\
\hline $24 \times 6$ & 0,855 & 0,139 & 0,945 & 0,946 & 0,071 & 0,944 \\
\hline $26 \times 6$ & 0,915 & 0,143 & 0,798 & 0,950 & 0,071 & 0,787 \\
\hline $28 \times 6$ & 0,904 & 0,145 & 0,736 & 0,930 & 0,090 & 0,983 \\
\hline Média & 0,914 & 0,108 & 0,876 & 0,927 & 0,082 & 0,893 \\
\hline
\end{tabular}

Louie \& Selker (2000) também encontraram, no seu procedimento de interpolação de perfis radiais de distribuição de água, valores maiores que 0,85 de coeficiente de determinação. Quanto aos valores de coeficiente de variação e à probabilidade de igualdade pelo teste $t$ de Student entre duas amostras, respectivamente, Molle \& Gat (2000) consideram adequados valores menores que 0,30 e maiores que 0,90 . Desta forma se verifica, nas Tabelas 1 e 2, que todos os valores de coeficiente de variação foram inferiores a $15 \%$ e os valores médios de probabilidade de igualdade, pelo teste $t$ de Student, dos perfis medidos e gerados por interpolação, se mantiveram próximos a $90 \%$.

Para utilização em aplicativos computacionais como o Simulasoft (Prado, 2004), os perfis radiais adimensionais de distribuição de água apresentados nas Figuras 1 e 2, devem ser transformados na sua forma dimensional multiplicandose os valores de distâncias adimensionais ao aspersor pelo raio de alcance e os valores de intensidades adimensionais de precipitação do aspersor pela intensidade média de aplicação de água. No procedimento de interpolação proposto por Louie \& Selker (2000) apenas é empregada à relação entre os valores de vazão do aspersor, na condição a ser interpolada e na condição na qual se conhece o perfil radial. Em algumas situações este procedimento pode gerar valores imprecisos para a distância ao aspersor visto que o comportamento, em função da pressão

Tabela 2. Valores de coeficientes de determinação $\left(R^{2}\right)$, coeficientes de variação $(C V)$ e probabilidades de igualdade $(\mathrm{P}(\mathrm{t}))$ pelo teste $\mathrm{t}$ de Student, observados nas interpolações do perfil radial entre bocais

\begin{tabular}{|c|c|c|c|c|c|c|c|c|c|}
\hline $\begin{array}{c}\text { Pressão } \\
\text { (kPa) }\end{array}$ & \multicolumn{9}{|c|}{ Bocais (mm) } \\
\hline 294 & 0,862 & 0,129 & 0,702 & 0,956 & 0,088 & 0,967 & 0,875 & 0,194 & 0,989 \\
\hline 490 & 0,965 & 0,065 & 0,894 & 0,934 & 0,061 & 0,833 & 0,973 & 0,052 & 0,977 \\
\hline 588 & 0,978 & 0,051 & 0,899 & 0,890 & 0,098 & 0,708 & 0,924 & 0,084 & 0,872 \\
\hline 686 & 0,923 & 0,090 & 0,847 & 0,893 & 0,120 & 0,897 & 0,843 & 0,119 & 0,836 \\
\hline
\end{tabular}


de serviço e do diâmetro de bocais, para o raio de alcance e vazão muitas vezes não é o mesmo.

Na Figura 3 é apresentado o efeito das interpolações entre pressões de serviço (Figura 3A) e entre bocais principais (Figura 3B) na forma dimensional do perfil radial de distribuição de água. O procedimento de dimensionalização do perfil radial apresentado na Figura 3 foi efetuado a partir das expressões de vazão (Eqs. 8, 9 ou 10) e de raio de alcance (Eqs. 11, 12 ou 13).

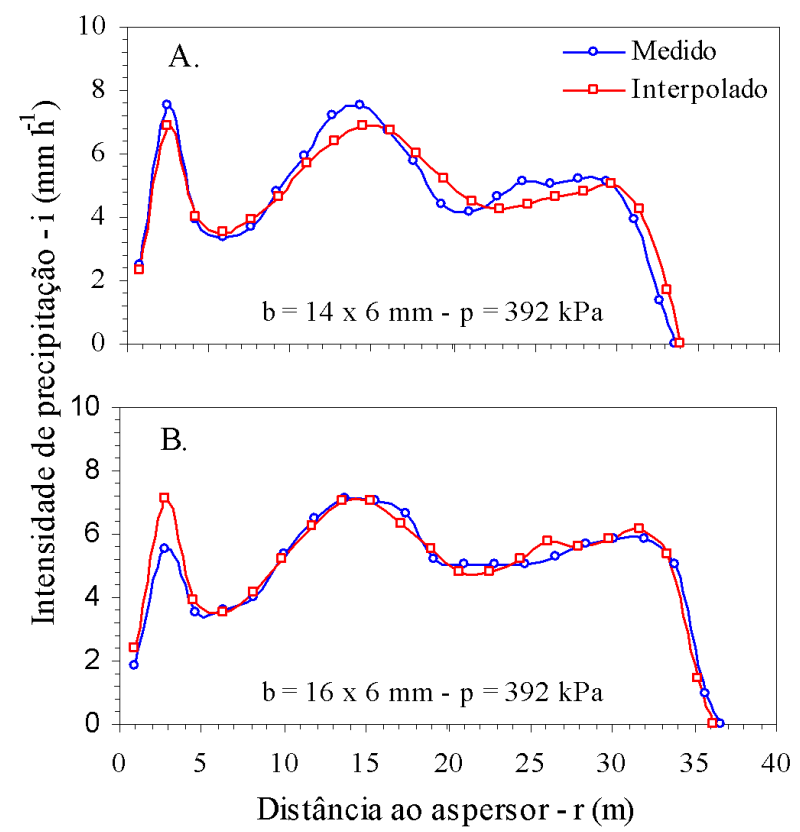

Figura 3. Perfis radiais na forma dimensional: observados e gerados pelo procedimento de interpolação entre pressões de serviço (A) e bocais (B)

Apresenta-se, na Figura 4, o comportamento dos valores do Coeficiente de Uniformidade de Christiansen (CUC) gerados em simulações digitais para sistemas autopropelidos de irrigação, com os perfis radiais obtidos por interpolação da pressão de serviço (Figura 4A) ou diâmetro bocal principal (Figura 4B) frente aos valores simulados com os perfis medidos em laboratório; ainda se observa, nesta figura, que as equações de reta que relacionam os valores do CUC, gerados com os perfis radiais obtidos em laboratório versus x perfis interpolados, apresentam coeficientes de determinação próximos à unidade e ficaram muito perto da reta 1:1. Os resultados apresentados na Figura 4 demonstram a adequação da metodologia de interpolação proposta para obtenção de perfis radiais intermediários.

A consistência dos perfis radiais interpolados para diferentes pressões de serviço foi evidenciada pelos pequenos erros absolutos observados (Figura 5A) que resultaram em um erro médio absoluto de $1,36 \%$ e um erro máximo de 5,04\%. Da mesma forma, a consistência dos perfis interpolados para diferentes diâmetros de bocais (Figura 5B) também foi evidenciada pelos pequenos erros absolutos observados que resultaram em um erro médio absoluto de 1,44\% com um erro máximo de 5,35\%.

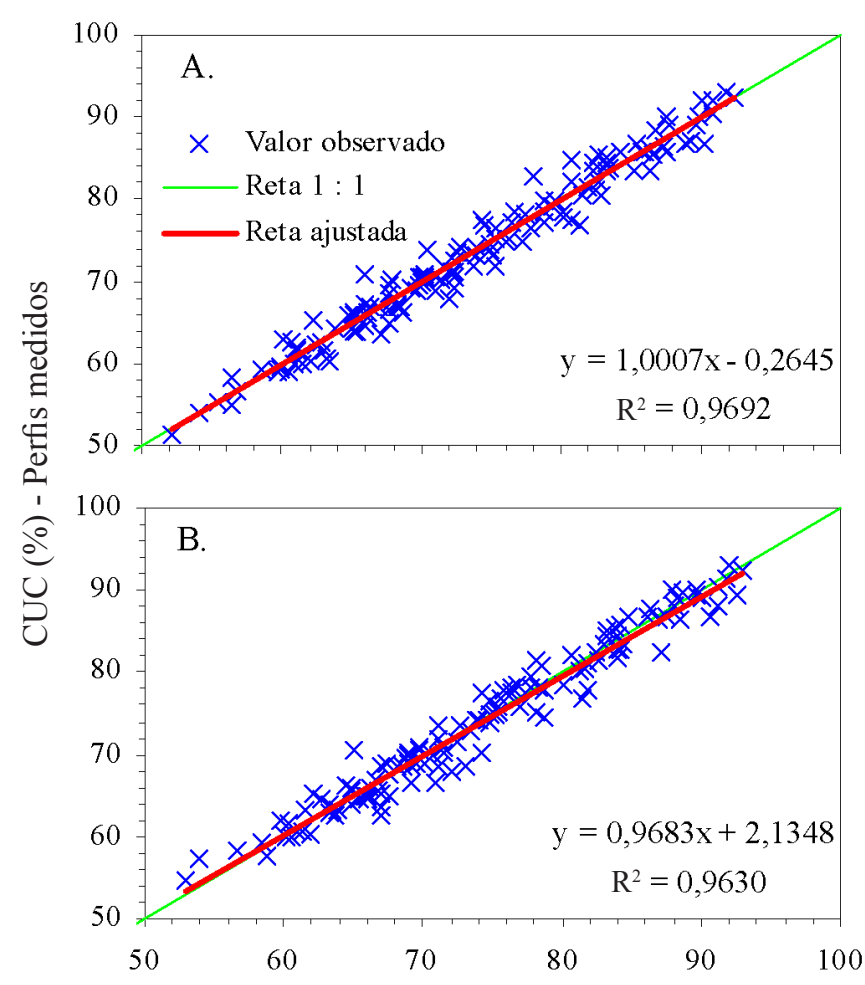

CUC (\%) - Perfis interpolados

Figura 4. Coeficientes de Uniformidade de Christiansen (CUC) simulados com perfis radiais interpolados entre pressões de serviço (A) ou entre bocais (B) versus simulados com perfis radiais medidos

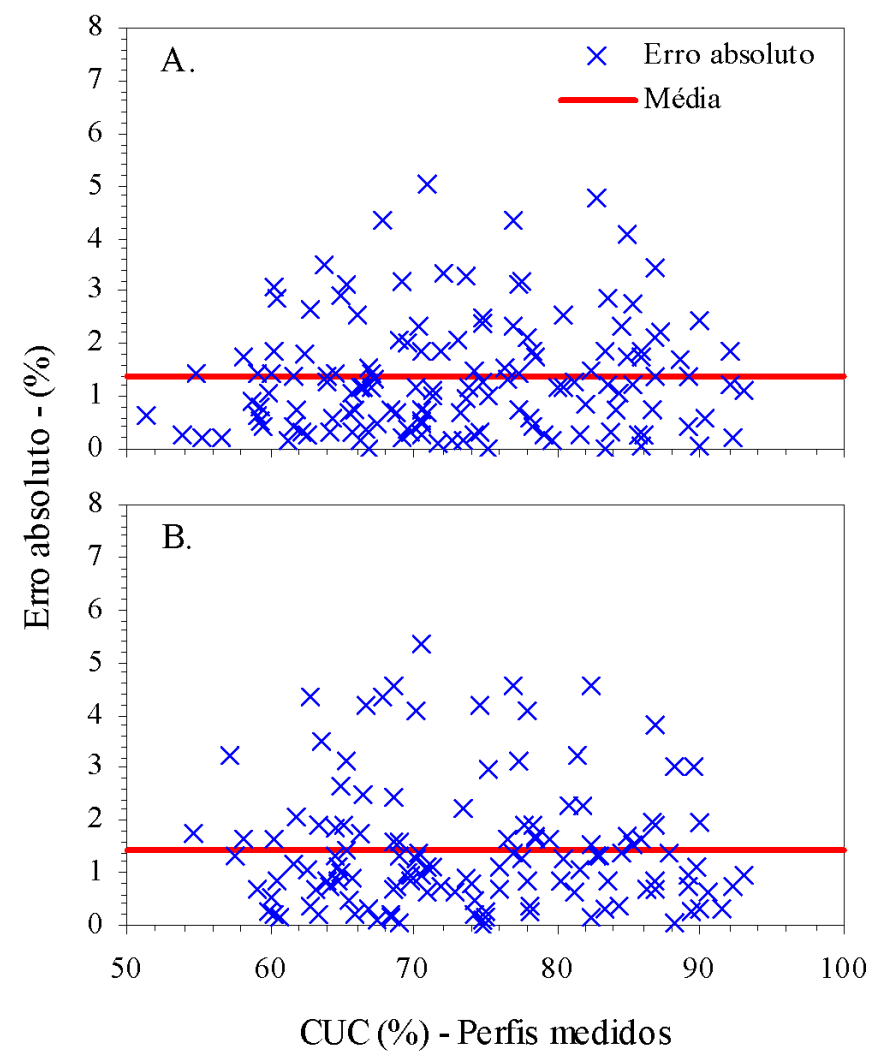

Figura 5. Erro absoluto no Coeficiente de Uniformidade de Christiansen (CUC) observado nas interpolações do perfil radial entre pressões de serviço (A) e bocais (B) 


\section{CONCLusões}

1. A metodologia proposta é adequada para interpolação de perfis radiais adimensionais.

2. O procedimento de interpolação requer apenas dois perfis radiais próximos para interpolar os valores de intensidade de precipitação adimensional do perfil radial, para pressão de serviço ou diâmetro do bocal desejado.

3. As interpolações não influenciaram, de forma significativa, os valores de uniformidade de aplicação de água de equipamentos autopropelidos de irrigação.

\section{Literatura Citada}

Álvarez, J. F. O.; Benito, J. M. T. M.; Valero, J. A. J.; Pérez, P. C. Uniformity distribution and its economic effect on irrigation management in semiarid zones. Journal of Irrigation and Drainage Engineering, v.130, p.257-268, 2004.

Bernardo, S.; Soares, A. A.; Mantovani, E. C. Manual de Irrigação. 7.ed. Viçosa: Imprensa Universitária, 2005. 611p.

Carrión, P.; Tarjuelo, J. M.; Monteiro, J. SIRIAS: A simulation model for sprinkler irrigation. Part I: Description of model. Irrigation Science, v.20, p.73-84, 2001.

Faria, L. C.; Colombo, A.; Oliveira, H. F. E.; Beskow, S.; Prado, G. Distorção do vento na área molhada por canhões hidráulicos: extensão da modelagem para aspersores médios. Revista Brasileira de Engenharia Agrícola e Ambiental, v.16, p.699-705, 2012.

Faria, L. C.; Colombo, A.; Oliveira, H. F. E.; Prado, G. Simulação da uniformidade da irrigação de sistemas convencionais de aspersão operando sob diferentes condições de vento. Engenharia Agrícola, v.29, p.19-27, 2009.

Louie, M.; Selker, J. S. Sprinkler head maintenance effects on water application uniformity. Journal of Irrigation and Drainage Engineering, v.126, p.142-148, 2000.

Molle, B.; Gat, Y. L. Model of water applications under pivot sprinkler. II Calibration and results. Journal of Irrigation and Drainage Engineering, v.126, p.348-354, 2000.
Oliveira, H. F. E.; Colombo, A.; Faria, L. C. Modelagem dos efeitos do vento sobre as dimensões do alcance do jato de um canhão hidráulico. Revista Brasileira de Engenharia Agrícola e Ambiental, v.13, p.818-824, 2009.

Oliveira, H. F. E.; Colombo, A.; Faria, L. C., Prado, G. Efeitos da velocidade e da direção do vento na uniformidade de aplicação de água de sistemas autopropelidos. Revista de Engenharia, v.32, p.669-678, 2012.

Prado, G. Aplicativo computacional para simulação da distribuição de água pelo aspersor PLONA-RL300 em sistemas autopropelidos de irrigação. Lavras: UFLA, 2004. 86p. Dissertação Mestrado

Prado, G.; Colombo, A. Caracterização técnica do aspersor PLONA-RL300. Irriga, v.10, p.53-63, 2005.

Prado, G.; Colombo, A. Composição de perfis radiais de distribuição de água de aspersores. Irriga, v.14, p.41-53, 2009.

Prado, G.; Colombo, A. Distribuição espacial da água aplicada por equipamentos autopropelidos de irrigação - Parte I: Modelagem com o Simulasoft. Irriga, v.15, p.51-62, 2010a.

Prado, G.; Colombo, A. Determinação do perfil radial de aspersores a partir de ensaios de distribuição de água em sistemas autopropelidos de irrigação. Engenharia Agrícola, v.30, p.232-243, 2010 b.

Prado, G.; Colombo, A. Ajuste de parâmetros para distorção da distribuição de água aplicada por canhões hidráulicos em condições de vento. Irriga, v.16, p.52-69, 2011.

Rocha, F. A.; Pereira, G. M.; Rocha, F. S.; Silva, J. O. Análise da uniformidade de distribuição de água de um equipamento autopropelido. Irriga, v.10, p.96-106, 2005.

Solomon, K.; Bezdek, J. C. Charactering sprinkler distribution patterns with a clustering algorithm. Transactions of American of Agricultural Engineers, v.23, p.899-906, 1980.

Vories, E. D.; Asce, S. M.; Bernuth, R. D. von. Simulating sprinkler performance in wind. Journal of Irrigation and Drainage Engineering, v.113, p.119-130, 1987. 